\title{
Integrated iBeacon/PDR Indoor Positioning System Using Extended Kalman Filter
}

\author{
Hengrui Zhang 1, a, Qichang Duan 1, b, Pan Duan ${ }^{2, \mathrm{c}}$ and Bei Hu ${ }^{1,3, \mathrm{~d}}$ \\ ${ }^{1}$ College of Automation, Chongqing University, Chongqing 400044, China. \\ ${ }^{2}$ Nanan Power Supply Company, State Grid Chongqing Electric Power Company, Chongqing \\ 400060, China. \\ ${ }^{3}$ Chongqing University of Science and Technology, Chongqing 401331, China. \\ acCorresponding author: henryyncn@163.com, ${ }^{b} \mathrm{qc} \_\mathrm{d} @$ sina.com, ${ }^{\mathrm{c}} \mathrm{duanpancqu@gmail.com}$ \\ d86322218@qq.com
}

Keywords: indoor positioning, iBeacon, pedestrian dead reckoning, Extended Kalman Filter.

\begin{abstract}
Indoor positioning is a challenging task in location-based services (LBS). The basic requirements of the indoor positioning system are high accuracy, availability, and cost and energy efficiency. Apple's Bluetooth Low Energy (BLE) based iBeacon along with pedestrian dead reckoning (PDR) system meets the aforementioned requirements. For iBeacon based indoor positioning, path-loss model is adopted to calculate the distance between user and iBeacon, and the maximum likelihood estimate positioning method is proposed for positioning. In the PDR positioning system, the Mahony Attitude and Heading Reference System (AHRS) is adopted to calculate the attitude of smart phone, in order to improve the performance of heading inference. Because of the presence of error accumulation over time in PDR based positioning system, along with the fact that the iBeacon based positioning system is susceptible to disturbance, we proposed an integration algorithm for iBeacon and PDR using Extended Kalman Filter (EKF). Experiments indicates that the proposed method can achieve a meter-level precision.
\end{abstract}

\section{Introduction}

With the rapid development of information technology, especially the popularity of mobile Internet and mobile terminals like smart phones, location-based services (LBS) has brought great convenience to people's lives. For example, Google Maps, Uber and Yelp are typical applications of LBS. As the most fundamental and important part of LBS, localization technology has been extensively researched and the outdoor localization system called Global Positioning System (GPS) is widely accepted. However, the signal strength of GPS is not strong enough to penetrate walls of buildings especially for underground buildings, resulting in low accuracy of indoor positioning. Furthermore, there are a range of obstacles such as walls and people in the indoor situation, making it more difficult to improve the accuracy of positioning, while indoor localization requires higher accuracy than GPS. Therefore, indoor positioning is more challenging and is a relatively novel field of research.

Since Roy Want, et al. designed the first indoor positioning system Active Badge [[1]] based on infrared in 1992, multiple solutions of indoor positioning have been proposed, such as ultrasound, WiFi, Radio Frequency Identification (RFID), Ultra-wide band (UWB), geomagnetic field, Bluetooth Low Energy (BLE) etc. Apple Inc. launched BLE based iBeacon [[2]] protocol in WWDC 2013, which can be used for indoor positioning. And iBeacon is widely used because of low cost and low energy.

Indoor positioning systems rely on a number of metrics including Received Signal Strength Indicator (RSSI)[[3]], Time of Arrival (TOA)[[4]], Time Difference of Arrival (TDOA)[[5]], Angle of Arrival (AOA)[[6]] etc. According to these metrics, distance or angles between beacons (WiFi, iBeacons, ultrasound, UWB, etc.) and the positioning target can be calculated. Given the coordinates of beacons, the coordinate of the target can be calculated using trilateration. 
Fingerprint indoor positioning systems [[7]] usually rely on WiFi or Bluetooth, including two phases: offline training and online positioning. In the offline training phase, much data should be collected by measuring the RSSI of the radio wave. These data which is related to location will be used to generate the fingerprint database. In the online positioning phase, the RSSI of current location is measured by smart phones. Then extract features and match the fingerprint database to estimate the current location. The defect of fingerprint positioning is that it takes a lot of time and manpower to collect the fingerprint. Besides, the RSSI would change which requires maintenance engineers to collect RSSI and update the fingerprint database regularly.

Pedestrian dead reckoning (PDR) [[8]] positioning systems, based on accelerometer, gyroscope and magnetometer, which are built into a smart phone, is widely used in indoor positioning. PDR system involves step detection, stride length estimation and heading inference. Acceleration measurements are used for step detection and stride length estimation. A gyroscope and a magnetometer are two typical heading sensors, integrated with accelerometer by Attitude and Heading Reference System (AHRS), the heading direction of pedestrian can be inferred. When the starting position is known, the next position can be calculated through PDR algorithm. However, stride length estimation can't work well because of the low accuracy of smart phone sensors. Moreover, the error of positioning will accumulate over time.

Huang et al. proposed an indoor positioning method based on metameric white light sources and sub-pixels on a color image sensor [[9]]. They deployed 3 groups of metameric white light LED, whose RGB intensity ratios are different. Then capture images through the camera of smart phone, analyze the intensity ratios of sub-pixels to identify the respective metameric white light sources. This method achieved high accuracy in experiment environment, but the performance in practice is unknown. Zhang et al. presented a framework combining vision and lidar [[10]]. Lidar is used to measure distances precisely, then detect edge points to realize 3D-reconstruction of environment. This system is applied to indoor positioning in Microsoft Indoor Localization Competition - IPSN 2016, and won the first place of 3D positioning group. But the drawback of this method is that the lidar is extraordinarily expensive.

Having narrated the pros and cons of aforementioned methods, the aim of this paper is designing an accurate, real-time, cost and energy efficient indoor positioning system. We would focus on researching the integration of iBeacon and PDR, since an iBeacon that can be deployed easily is able to work immediately as long as it is powered by batteries.

\section{Ibeacon based indoor positioning}

An iBeacon is small BLE enabled device that use Apple's proprietary iBeacon protocol, and broadcast its message which include Universally Unique Identifier (UUID), Major value and Minor value. We can query the coordinates of beacons through UUID, along with the RSSI, the coordinate of the target is available.

\subsection{Path-loss Model of BLE Signal}

As we can collect the RSSI values from the beacons using our Android phone, the distance between target and beacon can be calculated based on path-loss model. Path-loss model is described by Kumar in [[11]] and given in Equation (1).

$$
R S S I=C-10 n \cdot \lg \left(d / d_{0}\right)
$$

In the equation, $C$ is reference RSSI value at $d_{0}, n$ represents path-loss factor related to the environment, $d$ is the distance between the user and the beacon, while $d_{0}$ represents the reference distance which is 1 meter in our case.

Preprocess of RSSI Value

Due to the instability of received signal intensity and the presence of measurement error, the RSSI value will appear random fluctuations, which demands digital filtering of the RSSI value to improve the positioning accuracy. In this paper, we use Gaussian filter to filter out abnormal values of RSSI. And 120 sets of RSSI data were collected in a fixed position, the scatter plot of these data is shown in Fig. 1 (a). In the figure, most points fall between $-70 \mathrm{dBm} \sim-55 \mathrm{dBm}$, and the distribution of RSSI approximates the Gaussian distribution, distribution fitting result is shown in Fig. 1 (b). 


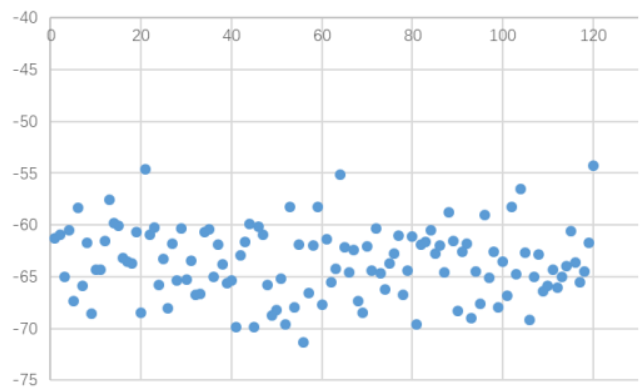

(a)

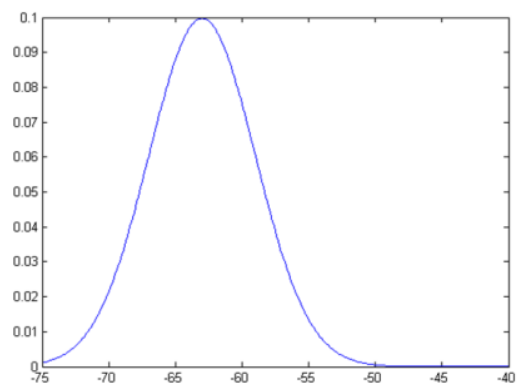

(b)

Figure 1. Scatter plot of RSSI values and Gaussian distribution fitting result.

The processes of Gaussian filtering are as follows: Given RSSI values, calculate the mean and variance as the coefficients $\mu$ and $\sigma^{2}$ of probability density function of Gaussian distribution, respectively, as shown in Equation (2) and (3).

$$
\begin{gathered}
\mu=\frac{1}{m} \sum_{i=1}^{m} X_{i}, \sigma^{2}=\frac{1}{m-1} \sum_{i=1}^{m}\left(X_{i}-\mu\right)^{2} \\
f(x)=\frac{1}{\sigma \cdot \sqrt{2 \pi}} e^{-\frac{(x-\mu)^{2}}{2 \sigma^{2}}}
\end{gathered}
$$

In the equation, random variable $X$ represents the RSSI values, its probability density function is $f(x)$. Then find the range of the random variable $\mathrm{X}\left[R S S I_{1}, R S S I_{2}\right]$ that makes $\mathrm{P}(\mathrm{X})>0.8$, i.e. $\int_{R S S I_{1}}^{R S S I_{2}} f(x) d x>0.8$. And the RSSI values that is not in the range of $\left[R S S I_{1}, R S S I_{2}\right]$ will be removed.

However, Gaussian filter cannot significantly impact on small fluctuations around the mean value. Thus, we use weighted sliding window filter to process the RSSI values, to minimize random fluctuations in small amplitude of the real-time signal. In practice, we found it is most efficient to set the window width as 4 and set weights as $0.5,0.2,0.2,0.1$, respectively. As is shown in Equation (4).

$$
R S S I_{N}^{*}=0.5 * R S S I_{N}+0.2 * R S S I_{N-1}+0.2 * R S S I_{N-2}+0.1 * R S S I_{N-3}
$$

Parameter Estimation of Path-loss Model using Least Square Method

Least square method is a common optimization algorithm, whose principle is to find the parameters that minimize the square sum of error. In order to get the parameters $C$ and $n$ in Equation (1), we put an iBeacon device in a fixed position and noted the preprocessed RSSI values on the Android phone for a number of distances. Suppose that the number of RSSI values is $m$, the actual distances between iBeacons and smart phone are $d_{1}, d_{2}, \cdots, d_{m}$. According to Equation (1), we suppose $b_{0}=C, b_{1}=n$, $X_{i}=-10 \lg \left(d_{i} / d_{0}\right), Y_{i}=R S S I_{i}$, then $Y_{i}=b_{0}+b_{1} X_{i}(i=1,2, \cdots, m)$. The loss function is:

$$
\text { Loss }=\sum_{i=1}^{m}\left(Y_{i}-b_{0}-b_{1} X_{i}\right)^{2}
$$

The optimal parameters $b_{0}^{*}$ and $b_{1}^{*}$ are as follows:

$$
\begin{gathered}
b_{0}^{*}=\frac{\sum X_{i}^{2} \sum Y_{i}-\sum X_{i} \sum X_{i} Y_{i}}{m \sum X_{i}^{2}-\left(\sum X_{i}\right)^{2}} \\
b_{1}^{*}=\frac{m \sum X_{i} Y_{i}-\sum X_{i} \sum Y_{i}}{m \sum X_{i}^{2}-\left(\sum X_{i}\right)^{2}}
\end{gathered}
$$

\subsection{Positioning Method Based on Maximum Likelihood Estimate}

Suppose that our smart phone received messages from $n$ beacons, and the coordinates of beacons are $\left(x_{1}, y_{1}\right),\left(x_{2}, y_{2}\right), \cdots,\left(x_{n}, y_{n}\right)$, respectively. With the help of aforementioned path-loss model, the distances $d_{1}, d_{2}, \cdots, d_{n}$ are known, as shown in Fig. 2. 


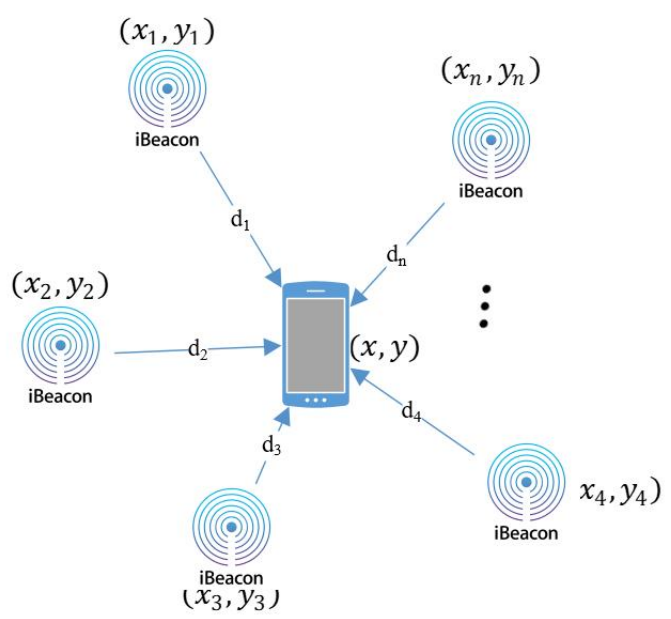

Then, we can get Equation (8) as follows:

Figure 2. Schematic for indoor positioning.

$$
\left\{\begin{array}{c}
\left(x_{1}-x\right)^{2}+\left(y_{1}-y\right)^{2}=d_{1}^{2} \\
\vdots \\
\left(x_{n}-x\right)^{2}+\left(y_{n}-y\right)^{2}=d_{n}^{2}
\end{array}\right.
$$

Equation (8) can be transformed into following format:

$$
\begin{gathered}
A X=b, X=\left[\begin{array}{l}
x \\
y
\end{array}\right] \\
A=\left[\begin{array}{cc}
2\left(x_{1}-x_{n}\right) & 2\left(y_{1}-y_{n}\right) \\
\vdots & \vdots \\
2\left(x_{n-1}-x_{n}\right) & 2\left(y_{n-1}-y_{n}\right)
\end{array}\right] \\
b=\left[\begin{array}{c}
x_{1}^{2}-x_{n}^{2}+y_{1}^{2}-y_{n}^{2}+d_{n}^{2}-d_{1}^{2} \\
\vdots \\
x_{n-1}^{2}-x_{n}^{2}+y_{n-1}^{2}-y_{n}^{2}+d_{n}^{2}-d_{n-1}^{2}
\end{array}\right]
\end{gathered}
$$

Based on maximum likelihood estimate, the coordinates of smart phone is $\hat{X}=\left(A^{T} A\right)^{-1} A^{T} b$.

\section{PDR based Positioning}

According to the PDR algorithm, accelerometer, gyroscope and magnetometer embedded in a smart phone will be combined into an inertial measurement unit to locate the position of the mobile terminal based on the following formula:

$$
\left\{\begin{array}{l}
X_{k+1}=X_{k}+S L_{k} \cos \theta_{k} \\
Y_{k+1}=Y_{k}+S L_{k} \sin \theta_{k}
\end{array}\right.
$$

Where $(X, Y)$ indicates the coordinates of the position, $S L$ is the stride length and $\theta$ represents the heading angle, thus the PDR algorithm mainly consists of the following steps including step detection, stride length estimation and heading inference.

\subsection{Step Detection and Stride Length Estimation}

Step detection and stride length estimation are based on the acceleration measurements of smart phone. We collected the 3-axis acceleration measurements of smart phone attached to a walking pedestrian, as is shown in Fig. 3 (a). It is obvious that the acceleration measurements of the three axes are periodic. To reduce the influence of the phone's orientation, the overall acceleration[[12]] is chosen to count steps. The overall acceleration described as follows:

$$
a=\sqrt{a_{x}^{2}+a_{y}^{2}+a_{z}^{2}}
$$

Where $a_{x}, a_{y}$ and $a_{z}$ are the acceleration of the three axes. The overall acceleration of 3 -axis acceleration measurements is shown in Fig. 3 (b). 


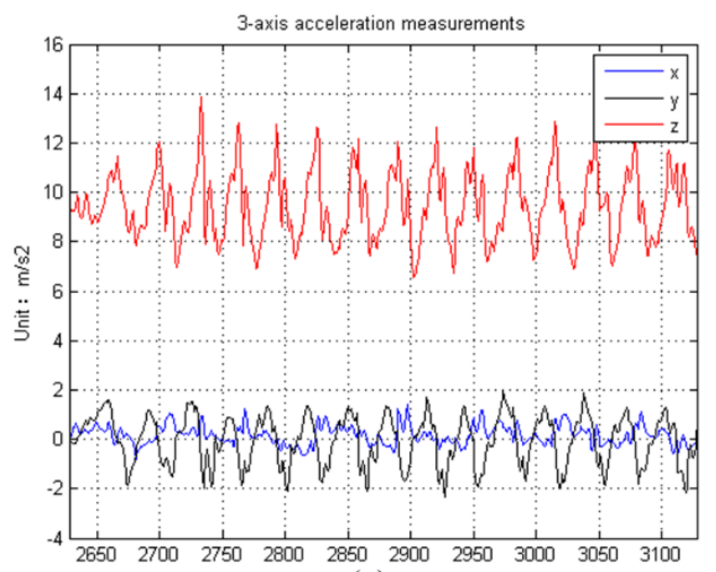

(a)

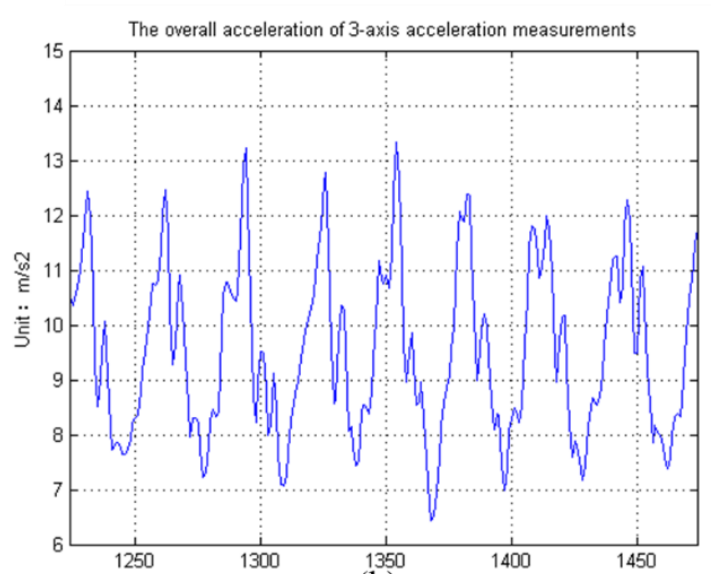

(b)

Figure 3. The 3-axis acceleration measurements (a) and the overall acceleration (b) for the accelerometer attached to a walking pedestrian.

In this paper, we adopted zero crossing detection method and improved the method for our situation. Firstly, a high-pass filter is used to eliminate the effects of gravitational acceleration. As the overall acceleration is multi-peak (as shown in Fig. 3), the sliding window averaging technique is utilized.

$$
a^{*}(k)=\frac{1}{N} \sum_{t=k-N+1}^{k} a(t)
$$

Where $\mathrm{N}$ denotes the window's size.

After sliding window averaging, the acceleration $a^{*}(k)$ will rise and cross the zero, reaching the peak; then decline and cross the zero, reaching the valley. Once the peak and valley of every step are available, we can plot the peak-valley curve and utilize zero crossing detection to count the steps (as shown in Fig. 4).

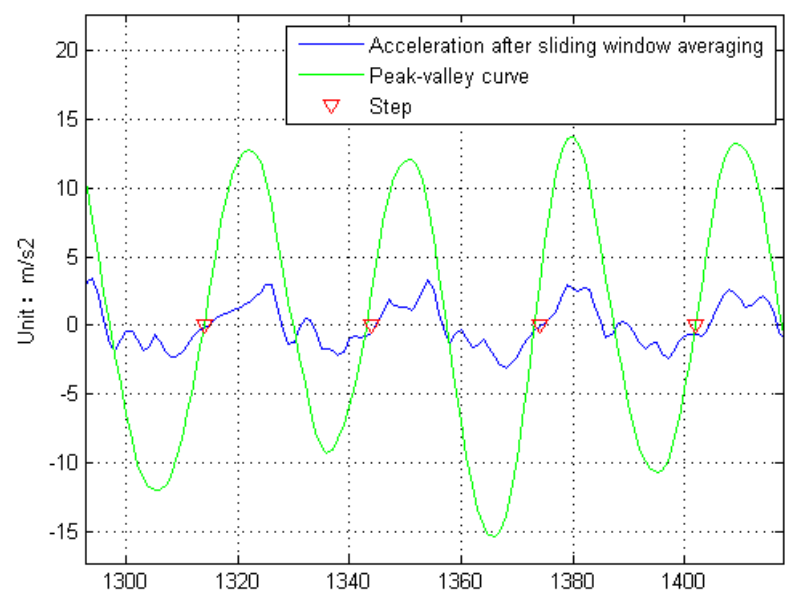

Figure 4. Zero crossing step detection.

Stride length estimation is an important factor that affects the positioning accuracy and can be performed in combination with a step detection procedure. The stride length is acceleration related and is typically given by:

$$
S L=k \sqrt[4]{a_{\text {peak }}-a_{\text {valley }}}
$$

Where $S L$ is the stride length of one step, $a_{\text {peak }}$ and $a_{\text {valley }}$ are the peak and valley value of the acceleration, respectively. The value of the coefficient $k$ depends on the individual and can be calibrated.

\subsection{Heading Inference}

Heading inference is a significant component of PDR based positioning. The heading angle $\psi$ is defined as the angle of rotation about the $\mathrm{z}$ axis of reference frame with respect to the horizon/ground. The measurements of accelerometer, gyroscope and magnetometer are based on body frame that is 
attached to smart phone, attitude calculation is necessary to rotate the body frame to reference frame. Attitude and Heading Reference System (AHRS) is a system that calculate the attitude and azimuth angle. In this paper, our AHRS algorithm is based on a complementary filter named Mahony filter[[13]]. The dynamic equation is given by:

$$
\dot{\boldsymbol{R}}=\boldsymbol{R} \boldsymbol{\Omega}_{\times}=\left[\begin{array}{ccc}
0 & -\omega_{z} & \omega_{y} \\
\omega_{z} & 0 & -\omega_{x} \\
-\omega_{y} & \omega_{x} & 0
\end{array}\right]
$$

Where $\boldsymbol{R}$ is the rotation matrix that describes the attitude of smart phone in reference frame. $\boldsymbol{\omega}=$ $\left[\omega_{x}, \omega_{y}, \omega_{z}\right]^{T}$ denotes the angular rate vector measured by gyroscope, which is unitized. In the body frame, geomagnetic vector $\boldsymbol{m}=\left[m_{x}, m_{y}, m_{z}\right]^{T}$ is measured by magnetometer, and acceleration vector $\boldsymbol{a}=\left[a_{x}, a_{y}, a_{z}\right]^{T}$ is measured by accelerometer, both of which are unitized. Transform $\boldsymbol{m}$ to reference frame as $\boldsymbol{m}_{\boldsymbol{r}}=\boldsymbol{R} \boldsymbol{m}$, and transform the unit vector of x-axis in body frame to reference frame as $\boldsymbol{c}=\boldsymbol{R} \boldsymbol{e}_{\boldsymbol{x}}$. The projection of $\boldsymbol{m}_{\boldsymbol{r}}$ and $\boldsymbol{c}$ in $x O y$ plane is given by:

$$
\boldsymbol{m}_{\boldsymbol{r}}^{\prime}=\boldsymbol{H} \boldsymbol{m}_{\boldsymbol{r}}, \boldsymbol{c}^{\prime}=\boldsymbol{H}, \boldsymbol{H}=\left[\begin{array}{lll}
1 & 0 & 0 \\
0 & 1 & 0
\end{array}\right]
$$

The AHRS algorithm is shown in Table 1 as follows.

TABLE 1. The procedure of AHRS algorithm.

\begin{tabular}{c|c}
$\begin{array}{c}\text { Steps } \\
1\end{array}$ & $\begin{array}{c}\text { Procedure } \\
\text { Get the initial } \boldsymbol{\omega}, \boldsymbol{m} \text { and } \boldsymbol{a} .\end{array}$ \\
\hline 3 & According to $\boldsymbol{m}$ and $\boldsymbol{a}$, get the attitude estimation $\boldsymbol{R}^{*}$ and the error vector \\
\hline 4 & Input $\boldsymbol{e}$ into PI controller, get the correction vector of angular rate $\delta \boldsymbol{\omega}$ \\
$\left(K_{p}+K_{I} * 1 / s\right) \boldsymbol{e}$.
\end{tabular}

Where the error vector $\boldsymbol{e}$ is given by:

$$
\boldsymbol{e}=\boldsymbol{a} \times\left(\boldsymbol{R}^{T} \boldsymbol{e}_{\mathrm{z}}\right)+\left|\boldsymbol{m}_{\boldsymbol{r}} \times \boldsymbol{c}^{\prime}\right| \boldsymbol{R}^{T} \boldsymbol{e}_{\mathrm{z}}
$$

Finally, transform rotation matrix $\boldsymbol{R}$ to Euler angles, adopt the azimuth angle as the estimation of heading angle $\psi$.

\section{4. iBeacon/PDR Integration Based on Extended Kalman Filter}

Because of the presence of error accumulation over time in PDR based positioning system, along with the fact that the iBeacon based positioning system is susceptible to disturbance, we proposed an integration algorithm for iBeacon and PDR.

The mathematical model of our system is given by the following dynamic equation (19) and observation equation (20).

$$
\boldsymbol{X}_{\boldsymbol{k}}=\left[\begin{array}{l}
x_{k} \\
y_{k} \\
\theta_{k}
\end{array}\right]=f\left(\boldsymbol{X}_{\boldsymbol{k}-\mathbf{1}}, \boldsymbol{u}_{\boldsymbol{k}-\mathbf{1}}\right)+\boldsymbol{W}_{\boldsymbol{k}-\mathbf{1}}=\left[\begin{array}{c}
x_{k-1}+\tilde{s} \cdot \cos \theta_{k-1} \\
y_{k-1}+\tilde{s} \cdot \sin \theta_{k-1} \\
\theta_{k-1}+\tilde{\theta}
\end{array}\right]+\boldsymbol{W}_{\boldsymbol{k}-\mathbf{1}}
$$

Where $x_{k}$ and $y_{k}$ are the position after k steps, $\theta_{k}$ is the heading direction after $k$ steps, $\boldsymbol{W}_{\boldsymbol{k}-\mathbf{1}}$ is three-dimensional system process noise, $\tilde{s}$ is the step length of step $k$, and $\tilde{\theta}$ is the variation of the heading direction in step $k$.

$$
\boldsymbol{Z}_{\boldsymbol{k}}=\left[\begin{array}{c}
x_{k} \\
y_{k} \\
s_{k} \\
\Delta \theta_{k} \\
\theta_{k}
\end{array}\right]=h\left(\boldsymbol{X}_{\boldsymbol{k}}\right)+\boldsymbol{V}_{\boldsymbol{k}}=\left[\begin{array}{c}
x_{k} \\
y_{k} \\
\sqrt{\left(x_{k}-x_{k-1}\right)^{2}+\left(y_{k}-y_{k-1}\right)^{2}} \\
\theta_{k}-\theta_{k-1} \\
\theta_{k}
\end{array}\right]+\boldsymbol{V}_{\boldsymbol{k}}
$$


Where $x_{k}$ and $y_{k}$ are the position of pedestrian from iBeacon based positioning, $s_{k}$ is the length of step $k$ from PDR positioning, and $\Delta \theta_{k}$ is the variation of the heading direction in step $k$ from the gyroscope, $\theta_{k}$ is the heading direction of step $k$ from the magnetometer, and $\boldsymbol{V}_{\boldsymbol{k}}$ is five-dimensional system measurement noise.

The principle of Extended Kalman Filter (EKF) is to carry out the first-order Taylor series expansion of the nonlinear model for the state estimation, then the classical Kalman Filter is available.

We linearized Equation (19) and (20) using first-order Taylor series expansion, and the result is as follows.

$$
\begin{aligned}
& X_{k}=A_{k, k-1} X_{k-1}+W_{k-1}+\varphi_{k-1} \\
& \boldsymbol{A}_{\boldsymbol{k}, \boldsymbol{k}-1}=\frac{\partial f}{\partial \widehat{\boldsymbol{X}}_{k-1}}=\left.\frac{\partial f\left(\widehat{\boldsymbol{X}}_{k-1}\right)}{\partial \boldsymbol{X}_{k-1}}\right|_{\boldsymbol{X}_{k-1}=\widehat{X}_{k-1}}, \boldsymbol{\varphi}_{k-1}=f\left(\widehat{\boldsymbol{X}}_{k-1}\right)-\left.\frac{\partial f}{\partial \boldsymbol{X}_{k-1}}\right|_{\boldsymbol{X}_{k-1}=\widehat{X}_{k-1}} \widehat{\boldsymbol{X}}_{k-1} \\
& Z_{k}=H_{k} X_{k}+V_{k}+y_{k} \\
& \boldsymbol{H}_{\boldsymbol{k}}=\left.\frac{\partial h}{\partial \widehat{\boldsymbol{X}}_{\boldsymbol{k}}}\right|_{\widehat{\boldsymbol{X}}_{\boldsymbol{k}, \boldsymbol{k}-1}}, \boldsymbol{y}_{\boldsymbol{k}}=h\left(\widehat{\boldsymbol{X}}_{\boldsymbol{k}, \boldsymbol{k}-1}\right)-\left.\frac{\partial h}{\partial \boldsymbol{X}_{\boldsymbol{k}}}\right|_{\boldsymbol{X}_{k, \boldsymbol{k}-1}} \widehat{\boldsymbol{X}}_{\boldsymbol{k}, \boldsymbol{k}-1}
\end{aligned}
$$

\section{Experiment and Result}

The positioning experiment was carried out in the corridor of 21st floor of the Main Teaching Building, Chongqing University. The iBeacons were deployed along the corridor in the test field. The testing device is Android phone OnePlus A0001. The transmitting power of our beacons is set to be $-12 \mathrm{dBm}$, and the transmitting frequency for iBeacon is set to be $1 \mathrm{~Hz}$.

The pedestrian carried the phone in his hands, walking along the corridor. The actual trajectory is a rectangle whose length and width are $20.8 \mathrm{~m}$ and $12 \mathrm{~m}$, respectively. We noted the iBeacon positioning results, PDR trajectory, and the iBeacon/PDR integration positioning trajectory. As is shown in Fig. 5.

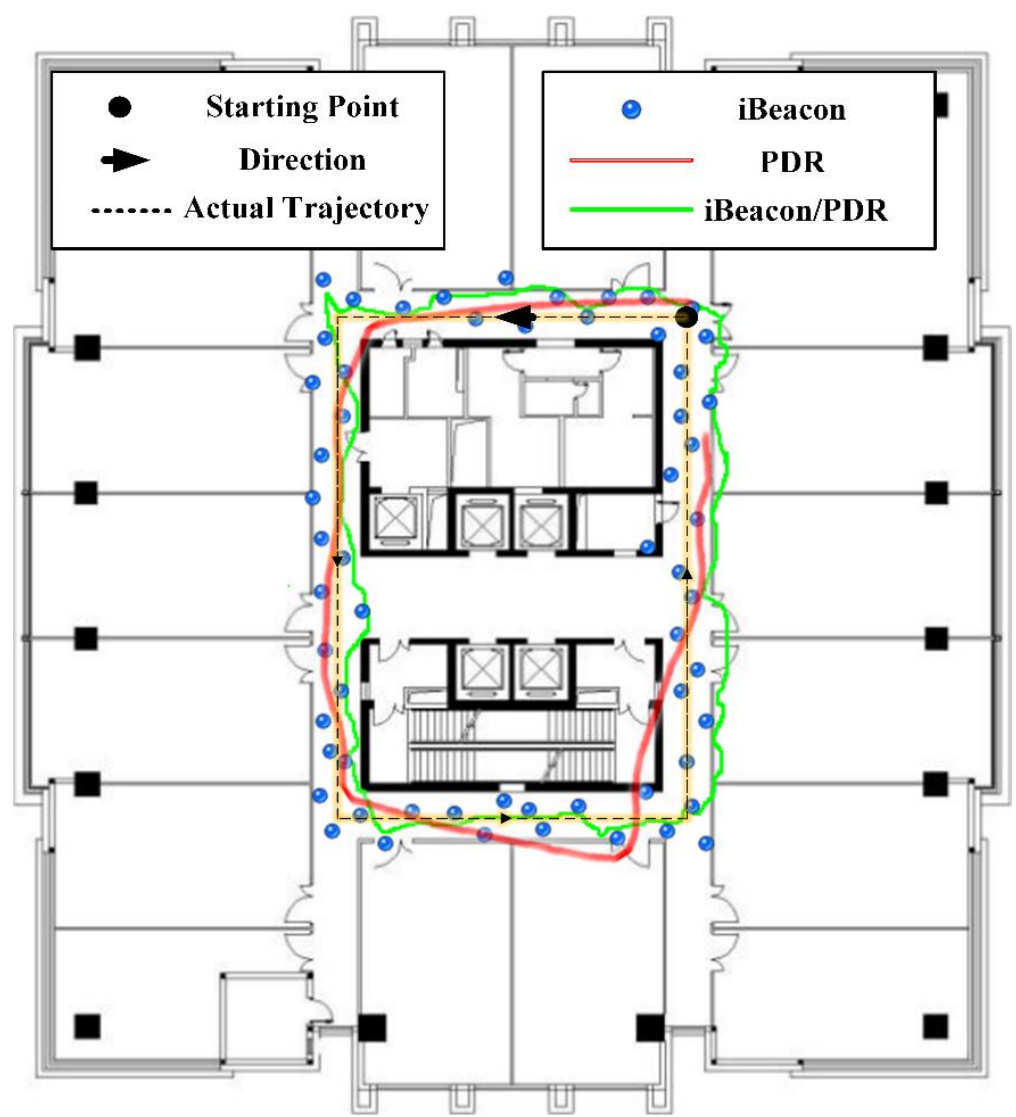

Figure 5. Experimental results.

In PDR experiment, the steps count of smart phone is 96, while the actual steps count is 98 . The accuracy of step detection is relatively high. However, the distance of PDR trajectory is $50.7 \mathrm{~m}$, compared to the actual distance $(65.6 \mathrm{~m})$, the error rate is $22.7 \%$, which is unsatisfactory. Having 
adopted iBeacon/PDR integration method, the distance of trajectory becomes $71.3 \mathrm{~m}$, the error rate declines to $8.7 \%$. Table 2 shows the statistics result of error for the three methods.

TABLE 2. Three Scheme comparing.

\begin{tabular}{|c|c|c|c|}
\hline & iBeacon & PDR & iBeacon/PDR \\
\hline Min. error/m & 0.4 & 1.3 & 0.5 \\
\hline Mean error/m & 2.1 & 6.7 & 1.3 \\
\hline Max. error/m & 3.8 & 14.9 & 5.7 \\
\hline
\end{tabular}

\section{Conclusion}

In this paper, we proposed an integrated iBeacon/PDR indoor positioning system that utilizes EKF to improve the positioning accuracy. For iBeacon based indoor positioning, the maximum likelihood estimate positioning method is proposed. In the PDR positioning system, the Mahony AHRS algorithm is adopted to calculate the attitude of smart phone, in order to improve the performance of heading inference. The experimental results indicate that the integration of iBeacon and PDR not only eliminated error accumulation over time in PDR, but also improved the positioning accuracy.

\section{References}

[1] Roy Want, et al. "The active badge location system." ACM Transactions on Information Systems (TOIS) 10.1 (1992), pp. 91-102.

[2] Information on https://developer.apple.com/ibeacon/Getting-Started-with-iBeacon.pdf

[3] Barsocchi, Paolo, et al. "A novel approach to indoor RSSI localization by automatic calibration of the wireless propagation model." Vehicular Technology Conference, 2009. VTC Spring 2009. IEEE 69th. IEEE, 2009.

[4] Guvenc, Ismail, and Chia-Chin Chong. "A survey on TOA based wireless localization and NLOS mitigation techniques." IEEE Communications Surveys \& Tutorials 11.3 (2009).

[5] Kim, Ryangsoo, et al. "TDOA localization for wireless networks with imperfect clock synchronization." Information Networking (ICOIN), 2014 International Conference on. IEEE, 2014.

[6] Tian, Hui, Shuang Wang, and Huaiyao Xie. "Localization using cooperative AOA approach." Wireless Communications, Networking and Mobile Computing, 2007. WiCom 2007. International Conference on. IEEE, 2007.

[7] Chen, Liang, et al. "Bayesian fusion for indoor positioning using Bluetooth fingerprints." Wireless personal communications (2013), pp. 1-11.

[8] Harle, Robert. "A Survey of Indoor Inertial Positioning Systems for Pedestrians." IEEE Communications Surveys and Tutorials 15.3 (2013), pp. 1281-1293.

[9] Huang, Heqing, et al. "Indoor Positioning Method Based on Metameric White Light Sources and Subpixels on a Color Image Sensor." IEEE Photonics Journal 8.6 (2016), pp. 1-10.

[10] Zhang, Ji, and Sanjiv Singh. "Visual-lidar odometry and mapping: Low-drift, robust, and fast." Robotics and Automation (ICRA), 2015 IEEE International Conference on. IEEE, 2015.

[11] Kumar, Praveen, Lohith Reddy, and Shirshu Varma. "Distance measurement and error estimation scheme for RSSI based localization in Wireless Sensor Networks." Wireless Communication and Sensor Networks (WCSN), 2009 Fifth IEEE Conference on. IEEE, 2009, pp. $1-4$.

[12] Chen, Guoliang, et al. "Integrated WiFi/PDR/Smartphone using an unscented kalman filter algorithm for 3D indoor localization." Sensors 15.9 (2015), pp. 24595-24614.

[13] Mahony, Robert, Tarek Hamel, and J-M. Pflimlin. "Complementary filter design on the special orthogonal group SO (3)." Decision and Control, 2005 and 2005 European Control Conference. CDC-ECC'05. 44th IEEE Conference on. IEEE, 2005. 